\title{
Investigando a Emergência de Relações Equivalentes a partir de Estruturas de Treino Mutuamente Excludentes
}

\author{
Paulo Roberto dos Santos Ferreira* (D), Vinícius Cabral Gonçalves, \\ Daiane Cristina Daleaste, \& Luciana Leonetti Correia \\ Universidade Federal da Grande Dourados, Dourados, MS, Brasil
}

\begin{abstract}
RESUMO - Investigou-se a formação de classes equivalentes a partir de estruturas de treino de discriminações condicionais que correspondem a relações emergentes mutuamente excludentes. Participaram um total de 10 universitários distribuídos em dois experimentos. As relações treinadas foram B1A1, B2A2, B3A3, D1C1, D2C2, D3C3, B1C2, B2C1, B3C3, D1A1, D2A2 e D3A3. Foram testadas as relações AC, CA, BD e DB. O Experimento II diferiu-se do Experimento I por incluir uma revisão das relações de treino. Os resultados sugerem a formação de classes para sete dos 10 participantes, sem diferenças significativas entre os experimentos. Contudo, foram estabelecidas diferentes classes para os participantes de ambos os experimentos, sugerindo que a ordem de treino não foi suficiente para determinar quais classes de equivalência seriam formadas.
\end{abstract}

PALAVRAS-CHAVE: equivalência de estímulos, classes excludentes, comportamento simbólico, controle de estímulos

\section{Investigating the Emergence of Equivalent Relationships with Mutually Exclusive Training Structures}

\begin{abstract}
This study investigated equivalence classes formation from conditional discrimination training structures that correspond to mutually exclusive emerging relationships. A total of 10 college students participated in two experiments. Trained relations were B1A1, B2A2, B3A3, D1C1, D2C2, D3C3, B1C2, B2C1, B3C3, D1A1, D2A2 and D3A3. Relations $\mathrm{AC}, \mathrm{CA}, \mathrm{BD}$ and DB were tested. Experiment II differed from Experiment $\mathrm{I}$ in that it included a review of training relations. The results indicated class formation for seven of the 10 participants, without significant differences between the experiments. However, different classes were established for the participants of both experiments, suggesting that the training order was not enough to determine which equivalence classes would be formed.
\end{abstract}

KEYWORDS: stimulus equivalence, excluding classes, symbolic behavior, stimulus control

A formação de classes de estímulos equivalentes vem sendo estudada desde o experimento inicial de Sidman (1971) e com maior intensidade após a operacionalização do fenômeno proposta por Sidman e Tailby (1982). Investigações mais recentes têm demonstrado que o fenômeno da emergência comportamental é fundamental para uma interpretação satisfatória do comportamento simbólico (Bortoloti, Rodrigues, Cortez, Pimentel, \& de Rose, 2013; Dickins \& Dickins, 2001; Dickins, Bentall, \& Smith, 1993; Sidman \& Tailby, 1982; Smith, Dickins, \&
Bentall, 1996; Fields, Arntzen, Nartey, \& Eilifsen, 2012). Estudos desse tipo representam de forma contundente um empreendimento científico que é importante no tocante às suas implicações práticas e, também, criam condições para o desenvolvimento teórico da área em sua busca de um retrato mais compreensível das relações simbólicas.

O procedimento empregado em grande parte dos estudos de equivalência de estímulos (Garotti, de Souza, de Rose, Molina, \& Gil, 2000; Sidman \& Tailby, 1982; Sidman, 2000) consiste no estabelecimento, por meio de reforçamento

* E-mail: paulorobertosantosferreira@gmail.com

- Submetido: 14/07/2016; Revisado: 05/09/2017; Aceito: 08/01/2018. 
diferencial e em contexto de escolha de acordo com o modelo (MTS, matching-to-sample), de um número mínimo de discriminações condicionais relacionando três estímulos ou mais para constituir uma classe de estímulos equivalentes. Essas relações são denominadas de relações treinadas ou relações de linha de base. Subsequentemente, são realizados os testes das relações que, por não terem sido anteriormente treinadas, são denominadas de emergentes.

Sidman e Tailby (1982) descreveram as três propriedades que devem ser verificadas para que se qualifique uma classe de estímulos como equivalente. Desse modo, a partir do treino $\mathrm{AB}$ (conforme esse tipo de nomenclatura, A é o estímulo condicional e B é o estímulo discriminativo em uma tentativa de MTS) e BC, têm-se demonstradas as três propriedades em situações de teste: reflexividade (AA, BB, CC), simetria (BA, CB), transitividade (AC) e combinação de transitividade e simetria (CA). A esse respeito, a investigação dos parâmetros define a emergência das relações que demonstram as referidas propriedades e denota, por consequência, a formação de classes de estímulos equivalentes e das suas possibilidades de extensão interpretativa e prática, que são crescentes e relevantes para a literatura científica da Análise do Comportamento e de disciplinas científicas afins.

As elaborações interpretativas e tecnológicas que têm como objeto o ensino ou modificação do comportamento simbólico a partir dos estudos da área são fortalecidas pelo fato da abordagem ser estritamente operacional (Sidman, 2000; Sidman \& Tailby, 1982). Não basta considerar a demonstração de relações comportamentais entre palavras e referentes, mas é igualmente necessário que se identifique as variáveis que determinam a emergência das relações que não foram diretamente ensinadas (Sidman \& Tailby, 1982).

Quanto aos delineamentos de pesquisa que avaliam o estabelecimento do comportamento simbólico, alguns estudos têm sugerido a importância de se investigar a modificação das relações emergentes estabelecidas por meio de procedimentos de MTS. Esses estudos visam descrever a reorganização das classes de estímulos formadas e tratam especialmente do papel da reversão de relações estabelecidas em linha de base ao alterarem as relações emergentes previamente testadas (Almeida \& Haydu, 2011; Pilgrim, Chambers, \& Galizio, 1995; Pilgrim \& Galizio, 1995).

Em um estudo seminal que lidou com o efeito da reversão de linha de base sobre a emergência comportamental, Pilgrim e Galizio (1995) treinaram as relações A1C1, A2C2, B1C1, B2C2, A1D1 e A2D2 e, subsequentemente, testaram as relações $\mathrm{CA}, \mathrm{CB}$ e $\mathrm{DA}$ (testes de simetria), e $\mathrm{AB}, \mathrm{BA}$, $\mathrm{BD}$ e $\mathrm{DB}$ (testes de transitividade e testes combinados de simetria e transitividade). A reversão se deu quando, na fase seguinte do estudo, os pesquisadores treinaram as relações A1D2 e A2D1, ou seja, implementaram a reversão de duas das relações previamente treinadas. Testes de emergência realizados posteriormente sugerem que a reversão teve efeitos proeminentes sobre as relações de simetria, mas não sobre as relações emergentes de transitividade. Por outro lado, Garotti et al. (2000), ao replicarem Pilgrim e Galizio (1995), obtiveram a reversão de ambos os tipos de relações emergentes (simetria e transitividade).

Portanto, os estudos que trataram da reversão de relações previamente estabelecidas têm subsidiado uma apreensão da formação de classes equivalentes como um processo complexo e mutável no comportamento individual, tornando a aplicação do paradigma de equivalência de estímulos (Sidman \& Tailby, 1982) mais promissora para o entendimento do comportamento simbólico que se estabelece em situações naturais. Embora um grande número dos símbolos que os indivíduos aprendem a relacionar faça parte do conjunto de relações significativas estáveis compartilhadas pelos membros de uma determinada comunidade, há também muitas relações simbólicas que se modificam durante a vida do indivíduo. Essa possibilidade de modificação de relações simbólicas previamente estabelecidas corresponde a um aspecto comportamental característico de pessoas cujo comportamento verbal e simbólico é adaptado em sua relação com uma determinada comunidade linguística (e.g., Carvalho \& de Rose, 2014). É sobre essa função das classes de estímulos equivalentes que se debruçam aqueles que investigam a reversão de relações equivalentes.

De modo geral, esse tipo de pesquisa tem demonstrado a possibilidade do treino de relações como A1B1, A2B2, $\mathrm{B} 1 \mathrm{C} 1, \mathrm{~B} 2 \mathrm{C} 2$, seguido de testes $\mathrm{AC}$ com verificação da emergência de $\mathrm{A} 1 \mathrm{C} 1$ e $\mathrm{A} 2 \mathrm{C} 2$ e, subsequentemente, do treino de A1B2 e A2B1, implicar a reversão das relações emergentes que passam a ser representadas por $\mathrm{A} 1 \mathrm{C} 2 \mathrm{e}$ A2C1. Com base nesse tipo de procedimento, deve ser considerada a importância da identificação de variáveis que determinam a modificação ou manutenção das relações de controle estabelecidas entre unidades comportamentais de diferentes classes de equivalência (Doran \& Fields, 2012; Mackay, Wilkinson, Farrell, \& Serna, 2011). Sendo assim, a reversão de relações de equivalência configura-se como um importante instrumento de investigação psicológica da transformação de significado.

Uma possibilidade adicional de estudo experimental sobre o tema da reversão de relações de equivalência, que tem sido muito pouco investigada, consiste no emprego de um tipo de treino com reversão simultânea entre as classes de estímulos, ao invés de reversão sucessiva, como foi utilizado por Pilgrim e Galizio (1995) em suas replicações sistemáticas. Conforme esse tipo de estrutura de treino, que ora denominamos como "estrutura excludente", as classes de equivalência delineadas em estudos de reversão seriam simultaneamente ensinadas. Essa estrutura define-se, portanto, como o treino de relações que levam à formação de classes excludentes ou incompatíveis com relação aos testes de emergência. A seguir, são apresentados os detalhes desse tipo de desenho experimental. 
Inicialmente, treina-se as relações $\mathrm{A} 1 \mathrm{C} 1, \mathrm{~A} 2 \mathrm{C} 2, \mathrm{~A} 3 \mathrm{C} 3$, B1D1, B2D2, B3D3, A1D2, A2D1, A3D3, B1C1, B2C2 e $\mathrm{B} 3 \mathrm{C} 3$, nessa ordem. A partir do emprego desse tipo de treino, testes das discriminações emergentes que representam as propriedades combinadas de simetria e transitividade envolvem dois pares de classes sobrepostas (A1B1C1 e A2B2C2 em contraposição a A1B2D2 e A2D1B1). Por essa razão, esse tipo de treino poderá esclarecer aspectos pouco explorados da relação entre estímulos pertencentes a diferentes classes de equivalência. Tais aspectos dizem respeito ao estabelecimento de classes de equivalência sobrepostas e, nessa abordagem do comportamento simbólico, de significados sobrepostos. Avaliar o efeito desse tipo de treino será útil em sua aplicação aos comportamentos semânticos sofisticados, como aqueles envolvidos na categorização, metáfora e abstração em contingências de quatro termos. Nesse sentido, a relação de referência suposta como base de uma teoria semântica experimentalmente corroborada pelas relações emergentes de equivalência não se restringiria a relações fixas e isoladas, estendendose a relações de significações múltiplas e polissêmicas, evidentemente mais condizente com o uso real da linguagem (Skinner, 1957; Ferreira, Domeniconi, \& de Rose, 2010).

Estudos anteriores (e.g., Folsta \& de Rose, 2007; Garotti \& de Rose, 2007; Pilgrim \& Galizio, 1995; Pilgrim et al., 1995) permitiram somente verificar a relação entre classes incompatíveis estabelecidas em sequência, por meio da reversão de relações previamente estabelecidas, e não simultaneamente, como no presente estudo. Diferentemente da presente investigação, os estudos realizados até então não investigaram a reversão simultânea envolvendo diferentes classes. A esse respeito, o tipo de treino empregado no presente estudo apresentou aspectos até então não investigados e permitiu a inédita avaliação de relações de equivalência entre classes simultaneamente incompatíveis. Dentre as implicações teóricas e práticas, destaca-se a possibilidade de se buscar uma definição de equivalência que possua uma maior generalidade científica, envolvendo relações potencialmente ambíguas tal como aquelas presentes na linguagem corrente, e a importância de se considerar a complexidade comportamental que caracteriza as relações simbólicas que se dão em contextos naturais, fora do laboratório.

Sendo assim, a partir da relevância de se investigar com maior precisão as variáveis envolvidas na interação entre classes equivalentes incompatíveis, o presente estudo foi constituído de dois experimentos que se diferenciaram pela adição da revisão de todas as relações de treino, anteriormente ao teste das relações, no segundo experimento. Os dois experimentos permitiram verificar se o treino de relações condicionais envolvidas na formação potencial de classes de equivalência incompatíveis estabeleceria consistentemente classes equivalentes e, em caso afirmativo, se os participantes apresentariam a formação das mesmas classes de equivalência.

\section{EXPERIMENTO ।}

O objetivo do Experimento 1 consistiu em treinar as relações B1A1, B2A2, B3A3, D1C1, D2C2, D3C3, B1C2, $\mathrm{B} 2 \mathrm{C} 1, \mathrm{~B} 3 \mathrm{C} 3, \mathrm{D} 1 \mathrm{~A} 1, \mathrm{D} 2 \mathrm{~A} 2, \mathrm{D} 3 \mathrm{~A} 3$ e, subsequentemente, testar as relações AC, CA, BD e DB.

\section{Método}

Participantes. Participaram do experimento seis estudantes universitários, de ambos os sexos, recrutados em cursos de graduação universitária, com idade entre 20 e 30 anos. Como critério de seleção, foi exigido que os participantes não houvessem participado de estudos de Psicologia Experimental, além de não terem conhecimento formal de Análise Experimental do Comportamento ou Lógica.

O estudo foi aprovado pelo Comitê de Ética em Pesquisa com Seres Humanos da própria universidade (conforme Parecer CONEP 827.415/2014).

Equipamentos e local. Foi utilizado um microcomputador para apresentar, com o emprego do software Superlab ${ }^{\circledR}$ (Cedrus, Phoenix, Arizona), as tarefas de discriminação condicional programadas pelos pesquisadores, além de registrar as respostas (clicar com o mouse no estímulo apresentado na tela do computador) dos participantes.

O experimento foi realizado em uma sala do Laboratório de Psicologia Experimental da universidade, na qual os participantes ficavam acomodados em cadeiras e respondiam aos estímulos mostrados pelo computador que, por sua vez, ficava sobre uma mesa próxima aos participantes. Durante a execução do procedimento, o pesquisador ficava na sala ao lado, aguardando ser chamado pelo participante após o término de cada sessão.

Procedimento. Os estímulos apresentados nas tarefas de discriminação condicional foram figuras abstratas retiradas de um conjunto de 12 figuras distintas, sendo cada qual designada alfanumericamente (A1, A2, A3, B1, B2, B3, C1, C2, C3, D1, D2 e D3). A Figura 1 apresenta esquematicamente os estímulos empregados e as relações treinadas e testadas.

A tarefa de MTS se iniciava com a apresentação de uma figura no centro da metade superior da tela, o estímulo modelo (estímulo condicional). Após a resposta de clicar no estímulo modelo apareciam simultaneamente outras três figuras, as escolhas (dentre as quais uma seria o estímulo discriminativo relacionado ao estímulo condicional), 


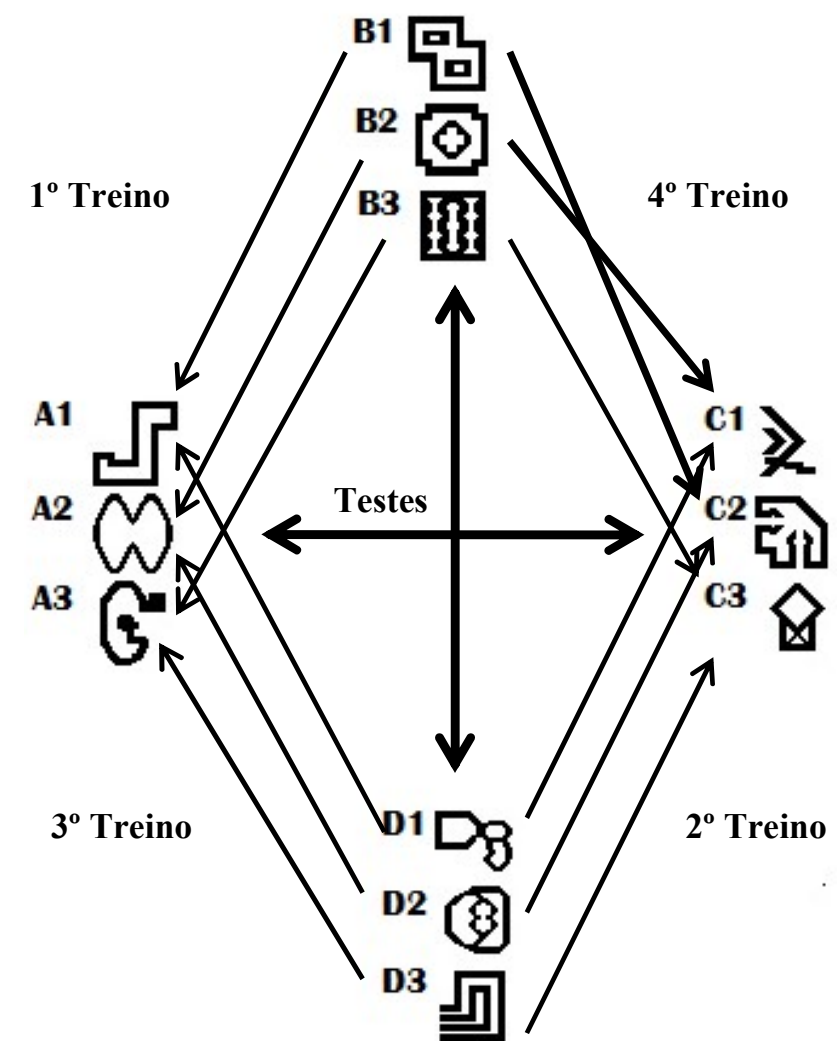

Figura 1. Estímulos visuais empregados nas tentativas de MTS simultâneo nos Experimentos 1 e 2 e ordem de treino e testes. As setas menos espessas indicam as relações treinadas e as setas mais espessas indicam as relações testadas. Ênfase para as relações B1C2 e B2C1 que compõem a inversão necessária para o estabelecimento da estrutura aqui denominada de "excludente."

distribuídas em três posições distintas na metade inferior da tela. O modelo e as três escolhas permaneciam na tela até que o participante clicasse em uma das escolhas, caracterizandose como procedimento de MTS simultâneo.

Nas tentativas de treino, as respostas do participante eram imediatamente seguidas de uma tela na cor azul claro com a palavra "CORRETO", caso indicassem acerto, ou seguidas de uma tela preta, caso representassem erros. Após esse tipo de consequência diferencial, seguia-se uma nova tentativa ou o fim da sessão. Diferentemente, nas tentativas de teste, a resposta do participante não produzia consequências diferenciais, sendo seguida por uma nova tentativa ou o fim da sessão.

As tentativas de treino e teste foram agrupadas em blocos e arranjadas de tal forma que, em cada bloco, os estímulos exibidos como modelos apareciam o mesmo número de vezes, o estímulo de escolha correto não aparecia na mesma posição mais do que o equivalente a $1 / 3$ das tentativas do bloco e um determinado estímulo escolha era apresentado o mesmo número de vezes em cada uma das posições possíveis. A quantidade de tentativas nos blocos de treinos foi de 36, no mínimo, mas dependeram também do atendimento ao critério que foi sempre de 18 acertos consecutivos. A quantidade de tentativas nos blocos de testes foi de 72 .

As seguintes instruções foram mostradas, na tela do computador, no início do primeiro bloco de cada uma das fases:

Fase de Treinos - Clique com o mouse sobre a figura apresentada na posição superior da tela. Em seguida, outras três figuras serão apresentadas na porção inferior da tela. Clique sobre uma delas e procure descobrir as relações entre as figuras. O computador informará se você acertou. Se você errar, aparecerá uma tela preta. Procure acertar! Boa sorte!! (Clique para começar).

Fase de Testes - Continue relacionando as figuras da mesma forma que nas etapas anteriores. Nesta etapa, entretanto, o computador não informará se você acertou ou errou. Procure acertar! Boa sorte! (Clique para começar).

Nos blocos de treino, o critério para o término da sessão e o seguimento para o próximo treino ou teste foi a realização de 36 tentativas, no mínimo, e acerto de 18 tentativas consecutivas. Os blocos de teste (ACCA e BDDB) consistiram em 72 tentativas que contemplaram todas as possibilidades combinatórias compreendendo os estímulos modelo e as posições dos estímulos de escolha. Nos blocos de treino foram treinadas diversas relações condicionais, conforme a ordem: B1A1, B2A2, B3A3; D1C1, D2C2, D3C3; B1C2, B2C1, B3C3; D1A1, D2A2, D3A3. Em seguida, foram testadas as relações emergentes na seguinte

Tabela 1

Fases do Experimento 1, critério de aprendizagem e relações treinadas/testadas

\begin{tabular}{lcc}
\hline Fase experimental & Critério & Relações treinadas/testadas \\
\hline 1 - Treino BA & 18 acertos consecutivos & B1A1, B2A2, B3A3 \\
2 - Treino DC & 18 acertos consecutivos & D1C1, D2C2, D3C3 \\
3 - Treino BADC & 18 acertos consecutivos & B1A1, B2A2, B3A3, D1C1, D2C2, D3C3 \\
4 - Treino BC & 18 acertos consecutivos & B1C2, B2C1, B3C3 \\
5 - Treino DA & 18 acertos consecutivos & D1A1, D2A2, D3A3 \\
6 - Treino BCDA & 18 acertos consecutivos & B1C2, B2C1, B3C3, D1A1, D2A2, D3A3 \\
7 - Teste ACCA & Sem critério & AC, CA \\
8 - Teste BDDB & Sem critério & BD, DB \\
\hline
\end{tabular}


ordem: AC, CA; BD, DB. A ordem dos blocos de treino e teste está sistematizada na Tabela 1 .

Foram empregados três critérios para a inferência de formação de classes de equivalência no estudo:

I) Considerando-se que para cada tentativa houve três alternativas, assumiu-se como critério de acerto acima do acaso o equivalente a mais do que um terço das respostas possíveis, ou seja, mais do que duas respostas corretas para cada relação no total de seis disponíveis;

II) Considerando-se que a concentração de respostas em apenas uma alternativa poderia simular a existência de uma relação condicional, assumiu-se como necessário o desempenho também na relação discriminativa concorrente. Ou seja, assumiu-se como necessário o responder consistente em ambas as relações, A1C1 e A2C2, para a inferência de formação de classes envolvendo esses estímulos;

III) Considerando-se que o paradigma proposto por Sidman e Tailby (1982) aponta a importância das relações de simetria e transitividade, assumiram-se, como critério, as relações emergentes simétricas. Ou seja, não bastaria a apresentação da relação $\mathrm{A} 1 \mathrm{C} 1$, sendo necessária também a apresentação de C1A1.

\section{Resultados e Discussão}

Fases de treino. Os seis participantes realizaram os Treinos de 1 a 6 (ver Tabela 1) e alcançaram o critério estabelecido, respondendo corretamente nas últimas 18 tentativas de cada sessão, conforme as contingências planejadas. Houve, contudo, uma diferença considerável entre os participantes no que diz respeito ao número de tentativas necessárias para a obtenção do critério (Tabela 2). O participante $\mathrm{P} 4$, por exemplo, precisou responder a 332 tentativas no Treino 1-BA, ao passo que o participante P1 obteve o critério em apenas 19 tentativas na sessão 4-BC.

Houve, em geral, uma dificuldade maior na realização de blocos com mais de seis discriminações condicionais (Treino 3-BADC e Treino 6-BCDA). Por exemplo, para quatro dos seis participantes, a sessão com maior número de tentativas para o atendimento do critério foi de treino combinado (P1=BADC; P2=BCDA; P5=BADC; P6=BCDA). Segundo
Saunders e Green (1999), tarefas com número maior de discriminações sucessivas demandam o aprendizado de um repertório discriminativo mais elaborado, o que explicaria essa diferença no número de tentativas necessárias nos diferentes blocos de treino.

Testes de emergência. Os resultados obtidos nos testes de emergência permitiram verificar quais classes de estímulos emergiram para cada participante. A estrutura de treino utilizada no experimento possibilitou, por meio dos quatro tipos de relações de emergência testados ( $\mathrm{AC}$, $\mathrm{CA}, \mathrm{BD}$ e DB), verificar se houve a formação das classes $\mathrm{ABC}, \mathrm{ABD}, \mathrm{ACD}$ ou BCD. Conforme mostra a Figura 2, houve uma discrepância entre os participantes em relação às classes formadas.

O participante $\mathrm{P} 1$ demonstrou a formação da classe ACD, conforme se verifica com a exibição das relações $\mathrm{A} 1 \mathrm{C} 1$, A2C2, C1A1 e C2A2. Os participantes P4 e P5 exibiram a emergência das relações, $\mathrm{A} 2 \mathrm{C} 1, \mathrm{~A} 1 \mathrm{C} 2, \mathrm{C} 1 \mathrm{~A} 2$ e $\mathrm{C} 2 \mathrm{~A} 1$, apresentando a formação da classe $\mathrm{ABC}$ que é excludente à classe $\mathrm{ACD}$.

Os participantes P1, P3 e P4 exibiram a emergência das relações B1D1, B2D2, D1B1 e D2B2, com formação da classe $\mathrm{ABD}$. Nenhum participante demonstrou a formação da classe $\mathrm{BCD}$, que é a classe excludente à $\mathrm{ABD}$, conforme se depreende do baixo desempenho no teste das relações B1D2, B2D1, D1B2 E D2B1 para os seis participantes.

O participante P6 e, em especial, o participante P2 apresentaram grande variabilidade nos resultados dos testes de emergência, não possibilitando atestar a formação de qualquer das classes de estímulos previstas pela estrutura. Por outro lado, quatro dos seis participantes (P1, P3, P4 e P5) apresentaram a formação de classes.

Os resultados dos testes das relações de emergência do Experimento 1 demonstraram a possibilidade de estabelecimento de classes de estímulos equivalentes a partir de uma estrutura de treino que envolve a formação potencial de classes mutuamente excludentes, assim definidas pelas contingências de reforço estabelecidas em tarefas de treino de discriminação condicional.

Não houve, no entanto, consistência entre os participantes no que diz respeito às classes que foram formadas. Uma vez que a ordem de treino foi a mesma para todos os participantes, essa variabilidade nas classes estabelecidas

Tabela 2

Tentativas necessárias para que cada participante alcançasse o critério nas fases de treino

\begin{tabular}{|c|c|c|c|c|c|c|}
\hline Participantes & 1-BA & 2-DC & 3-BADC & 4-BC & 5-DA & 6-BCDA \\
\hline $\mathrm{P} 1$ & 21 & 30 & 48 & 19 & 27 & 30 \\
\hline P2 & 33 & 36 & 60 & 42 & 18 & 90 \\
\hline P3 & 30 & 75 & 56 & 54 & 44 & 36 \\
\hline P4 & 332 & 51 & 36 & 102 & 24 & 126 \\
\hline P5 & 120 & 39 & 192 & 24 & 66 & 186 \\
\hline P6 & 33 & 18 & 108 & 51 & 36 & 174 \\
\hline
\end{tabular}



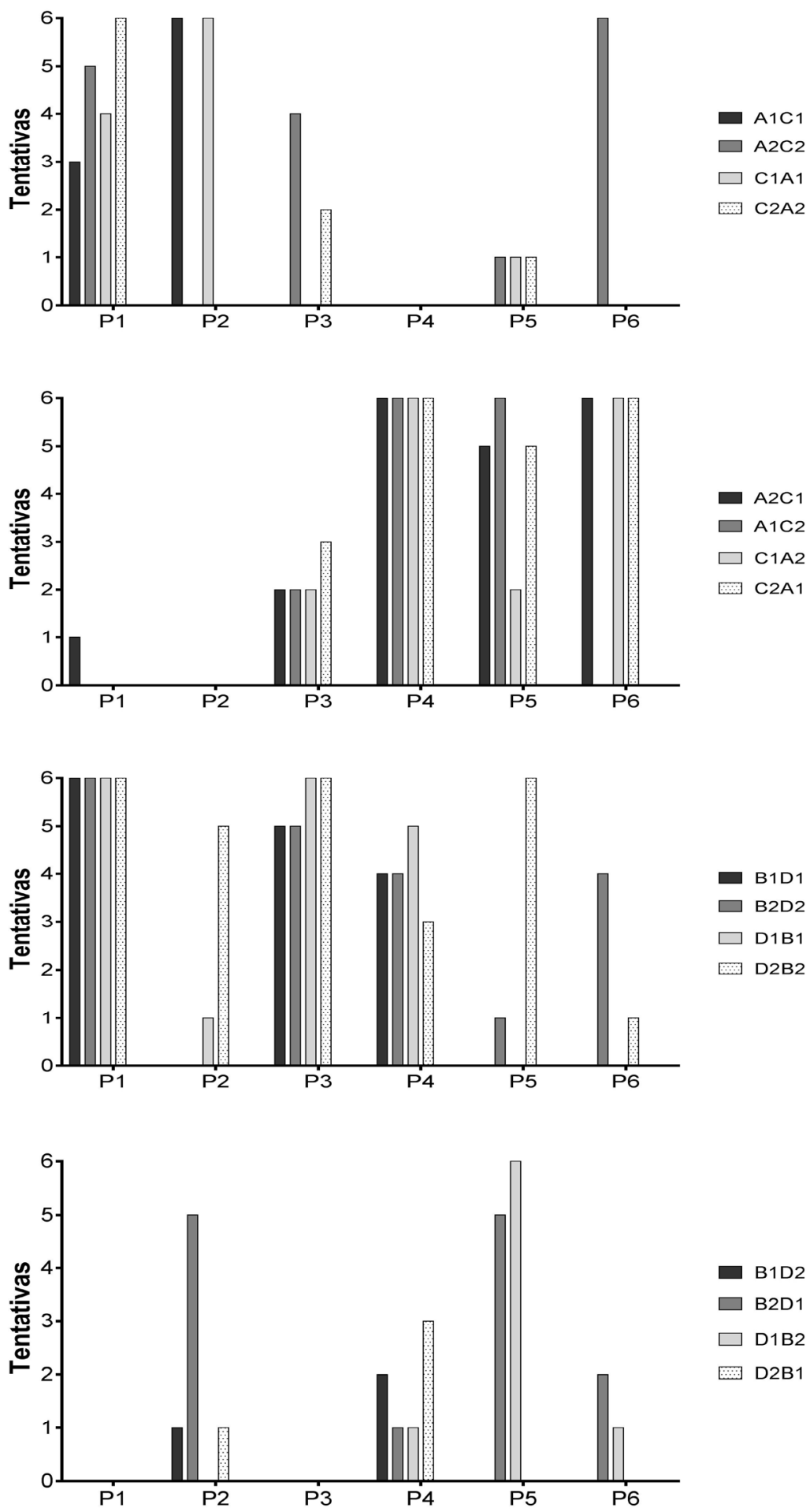

Figura 2. Número de escolhas das relações realizadas pelos participantes do Experimento 1 nas tentativas de emergência das sessões 7-ACCA e 8-BDDB.

sugere que a ordem de treino não foi suficiente para a especificação das relações que emergiram.

Os resultados do Experimento 1 apresentaram dois tipos de variabilidade. Em primeiro, variabilidade entre os participantes em relação ao tipo de classes de estímulos estabelecidas. Esse tipo de variabilidade poderia indicar que ocorreram nódulos (Fields, Adams, Verhave, \& Newman,
1990) diferentes de emergência para os participantes, a despeito de o treino seguir sempre uma mesma sequência experimental. Por exemplo, P1 relacionou consistentemente B1 com D1, e B2 com D2 nos testes de emergência, sugerindo uma consistência com o nódulo A, ao invés de B.

Um segundo tipo de variabilidade refere-se à inconsistência nos resultados dos testes de emergência 
para alguns dos participantes, sendo insuficientes para a demonstração de qualquer uma das duas classes potenciais. Esse tipo de variabilidade é ilustrado pelos resultados de P2 e P6 e pode ser uma decorrência do treino que implicou em relações fracamente estabelecidas nesse primeiro experimento. Uma alternativa interpretativa para a inconsistência na formação das classes reside na possibilidade de relações previamente treinadas terem sido deterioradas devido aos treinos subsequentes, o que impossibilitaria uma inferência sobre a variabilidade como intrínseca ao tipo de treino empregado. Visando verificar essa possibilidade é que se realizou o Experimento 2.

\section{EXPERIMENTO 2}

O Experimento 2 foi uma replicação sistemática do Experimento 1, diferindo deste apenas pela realização de uma revisão de todas as relações na fase 7-Treino BADCBCDA anteriormente à realização dos testes de emergência comportamental. $\mathrm{O}$ objetivo dessa revisão era garantir que todas as relações de treino fossem demonstradas imediatamente antes da realização dos testes de emergência. Dessa forma, permitiu-se averiguar se a variabilidade dos resultados entre os participantes foi um resultado da deterioração das relações de treino.

\section{Método}

Participantes. Participaram quatro estudantes universitários de ambos os sexos, recrutados em cursos de graduação da universidade, seguindo o mesmo critério de seleção empregado para o Experimento 1.

Equipamentos e local. Idem ao Experimento 1.

Procedimento. O Experimento 2, da mesma forma que o Experimento 1, consistiu na aplicação de tarefas de MTS realizadas pelos participantes no computador, segundo uma ordem e critérios de mudança de fases (Tabela 3 ). No entanto, o Experimento 2 contou adicionalmente com a fase de 7-Treino BADCBCDA que consistiu em uma revisão simultânea de todas as relações de linha de base, e que foi aplicada anteriormente à realização dos testes de emergência 8-Teste ACCA e 9-Teste BDDB.

\section{Resultados e Discussão}

Fases de treino. Os quatro participantes realizaram os blocos de treino (Treinos de 1 a 7 da sequência de fases experimentais, conforme a Tabela 3 ) e alcançaram o critério estabelecido, respondendo corretamente nas últimas 18 tentativas de cada sessão. Houve, como no Experimento 1, também uma diferença considerável entre os participantes no que diz respeito ao número de tentativas necessárias para a obtenção do critério, conforme os dados apresentados na Tabela 4. O participante P7, por exemplo, realizou 659 tentativas na sessão 7-BADCBCDA, ao passo que o participante P8 obteve o critério em 134 tentativas no mesmo tipo de sessão. Corroborando de forma mais clara um resultado obtido no Experimento 1, a sessão que exigiu um número maior de tentativas para todos os participantes foi a sessão com o maior número de relações, e, portanto, com o maior número de discriminações condicionais a serem aprendidas (Saunders, \& Green, 1999).

Testes de emergência. $O$ participante $P 9$ demonstrou a formação da classe $\mathrm{ACD}$, conforme se verifica com a exibição das relações A1C1, C1A1, A2C2 e C2A2. O participante P10 exibiu a emergência das relações, A1C2, C2A1 A2C1 e C1A2, demonstrando a formação da classe $\mathrm{ABC}$ que é excludente à classe $\mathrm{ACD}$ (Figura 3).

$O$ participante P10 exibiu a emergência das relações B1D1, B2D2, D1B1 e D2B2, demonstrando a formação da classe ABD. O participante $\mathrm{P} 7$ demonstrou a formação da

Tabela 3

Fases do Experimento 2, critério de aprendizagem e relações treinadas/testadas

\begin{tabular}{lcc}
\hline Fase experimental & Critério & Relações treinadas/testadas \\
\hline 1 - Treino BA & 18 acertos consecutivos & B1A1, B2A2, B3A3 \\
2 - Treino DC & 18 acertos consecutivos & D1C1, D2C2, D3C3 \\
3 - Treino BADC & 18 acertos consecutivos & B1A1, B2A2, B3A3, D1C1, D2C2, D3C3 \\
4 - Treino BC & 18 acertos consecutivos & B1C2, B2C1, B3C3 \\
5 - Treino DA & 18 acertos consecutivos & D1A1, D2A2, D3A3 \\
6 - Treino BCDA & 18 acertos consecutivos & B1C2, B2C1, B3C3, D1A1, D2A2, D3A3 \\
7 - Treino BADCBCDA & 18 acertos consecutivos & B1A1, B2A2, B3A3, D1C1, D2C2, D3C3, \\
8 - Teste ACCA & B1C2, B2C1, B3C3, D1A1, D2A2, D3A3 \\
9 - Teste BDDB & Sem critério & AC, CA \\
\hline
\end{tabular}


Tabela 4

Tentativas necessárias para que cada participante alcançasse o critério nas fases de treino.

\begin{tabular}{lccccccc}
\hline Participantes & 1-BA & 2-DC & 3-BADC & 4-BC & 5-DA & 6-BCDA & 7-BADCBCDA \\
\hline P7 & 129 & 34 & 67 & 41 & 18 & 139 & 627 \\
P8 & 53 & 81 & 62 & 67 & 23 & 51 & 134 \\
P9 & 19 & 108 & 43 & 75 & 30 & 142 & 659 \\
P10 & 34 & 26 & 56 & 109 & 24 & 92 & 255 \\
\hline
\end{tabular}
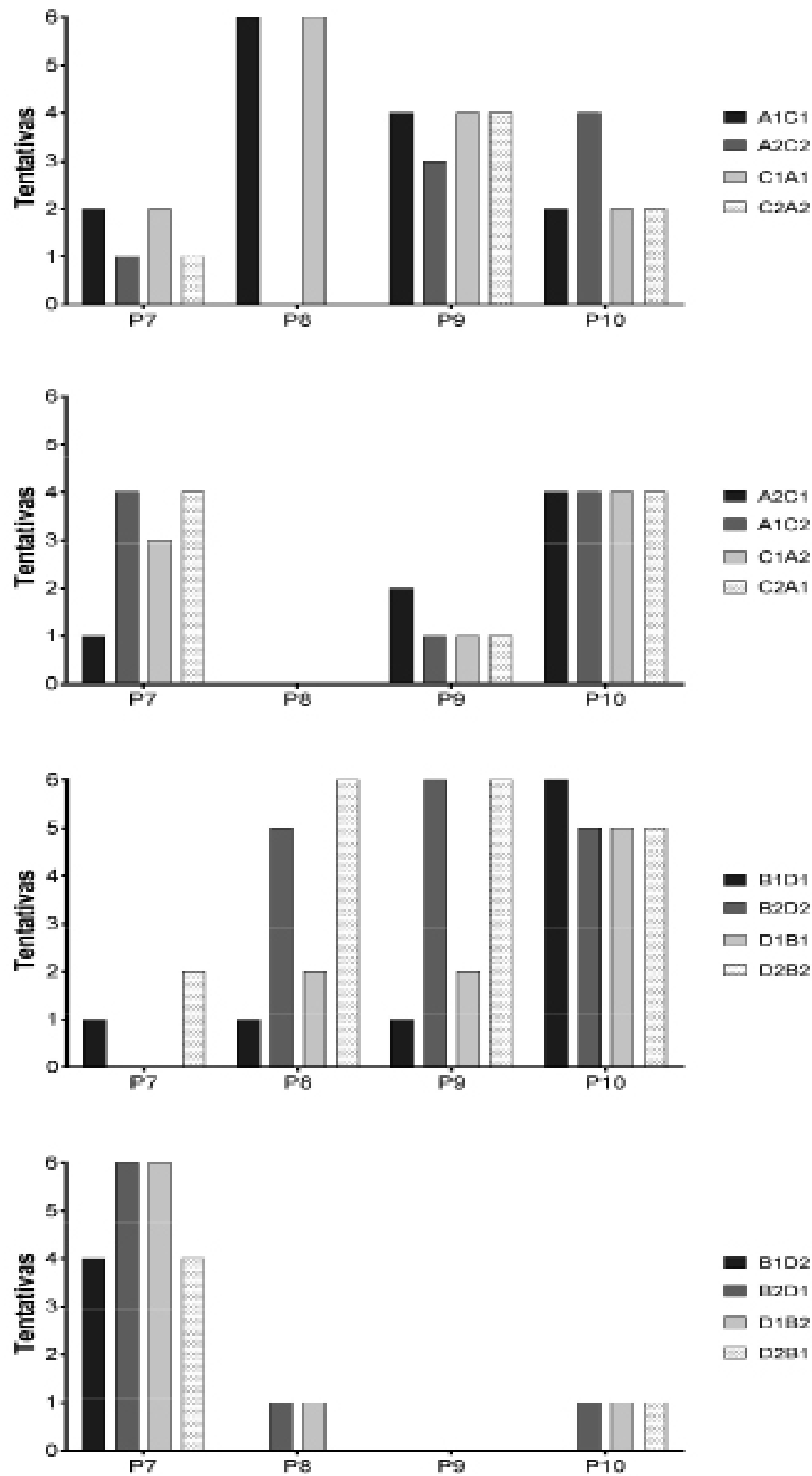

口ามเ

Figura 3. Número de escolhas das relações realizadas pelos participantes do Experimento 2 nas tentativas de emergência das sessões 7-ACCA e 8-BDDB. 
classe $\mathrm{BCD}$, que é a classe excludente à $\mathrm{ABD}$, conforme se depreendem do desempenho no teste das relações B1D2, B2D1, D1B2 e D2B1.

O participante P8 apresentou grande variabilidade nos resultados dos testes de emergência, não possibilitando atestar a formação de qualquer das classes de estímulos previstas pela estrutura. Por outro lado, três dos quatro participantes (P7, P9, P10) demonstraram a formação de classes.

Os resultados obtidos com os testes das relações de emergência do Experimento 2 acompanharam os dados obtidos no Experimento I. Da mesma forma que no Experimento 1, ficou evidente a formação de classes de estímulos para a maior parte dos participantes (três dos quatro). Ainda, os dois experimentos apresentaram o mesmo tipo de variabilidade entre os participantes no que diz respeito às classes que foram formadas. Os resultados do Experimento 2 sugerem que essas características, comuns aos resultados do Experimento 1, não decorreram de uma deterioração de relações de treino anteriormente às tarefas de emergência.

\section{DISCUSSÃO GERAL}

Todos os 10 participantes de ambos os experimentos alcançaram o critério de desempenho nas sessões de treino, a despeito das relações serem incompatíveis entre si, e sete deles apresentarem a formação de classes equivalentes. Os resultados replicam os obtidos por Garotti et al. (2000) mas também os estendem ao apresentarem a emergência de relações de transitividade consistentes com a reversão de relações simultaneamente incompatíveis. Nesse aspecto, o presente estudo acrescenta generalidade aos estudos da área. Ainda, os resultados sugerem que não foi a incompatibilidade entre as relações que representou uma dificuldade nos treinos, mas, sim, o número de discriminações condicionais, ou de discriminações sucessivas exigidas para o alcance do critério em sessões de treino com muitas relações (Saunders \& Green, 1999). A esse respeito, é importante considerar que a grande discrepância entre os participantes no número de tentativas necessárias para o término da fase de treino não implicou em diferenças consideráveis nos desempenhos em sessões de teste.

Interessantemente, foi grande a variabilidade a respeito de quais classes de estímulos equivalentes foram estabelecidas entre os participantes de ambos os experimentos. Os resultados sugerem que a ordem de treino não determina que classes de estímulos serão estabelecidas, uma vez que a sequência de treino foi a mesma entre os participantes. Esse dado é inédito e questiona estudos anteriores ao afirmarem a preponderância de algumas relações sobre outras pelo simples fato de terem sido estabelecidas previamente (e.g., Pilgrim \& Galizio, 1995). No entanto, é difícil estabelecer qual a variável que teria contribuído para a determinação das relações emergentes demonstradas nos testes AC, CA, $\mathrm{BD}$ e DB.

Uma possibilidade explicativa para esse tipo de variabilidade entre os participantes seria a de que diferentes nódulos das relações de emergência teriam se estabelecido com diferente "força" em função da quantidade de treino a que foram expostos os participantes. Garotti et al. (2000) propõem esse tipo de explicação para os seus resultados, segundo a qual a quantidade de reforçamento correlacionada (Nevin, 1992) com uma relação comportamental seria responsável pela preponderância dessa relação sobre outra concorrente, interferindo nos resultados de emergência. Retomando-se o que foi dito a respeito de $\mathrm{P} 1$, os seus resultados nos testes $\mathrm{BD}$ e DB sugerem a presença das relações B1D1 e B2D2, o que indicaria a emergência pelo nódulo $\mathrm{A}$, ao invés do nódulo $\mathrm{C}$. No entanto, a quantidade de tentativas realizadas para esse participante nas tarefas de treino envolvendo o nódulo $\mathrm{A}$ foi de 87 $(1-\mathrm{BA}=21 ; 3-\mathrm{BADC} / 2=24 ; 5-\mathrm{DA}=27,6-\mathrm{BCDA} / 2=15)$, contra 88 nas tarefas envolvendo o nódulo $\mathrm{C}(2-\mathrm{DC}=30$, $3-\mathrm{BADC} / 2=24,4-\mathrm{BC}=19,6-\mathrm{BCDA} / 2=15$ ), representando uma diferença insignificante na quantidade de tentativas (e de reforçamento) envolvendo os dois diferentes nódulos. Os resultados de P1 sugerem, portanto, que o tipo de explicação proposto por Garotti et al. (2000) não é suficiente para interpretar resultados como os obtidos no presente estudo.

Um segundo tipo de variabilidade apontado no estudo é o da inconsistência do responder para cada participante no que diz respeito à demonstração das classes equivalentes. Essa inconsistência foi, possivelmente, um resultado da ambiguidade inerente ao tipo de treino na sua relação com as relações emergentes que fossem consistentes com as relações estabelecidas em treino. Nesse sentido, é preciso considerar que em tentativas de teste, o participante poderia escolher entre alternativas diferentes e, mesmo assim, exibir desempenho condizente com determinadas relações treinadas. Por exemplo, em uma tentativa em que o modelo apresentado foi D1, o participante teria supostamente razões comparáveis para escolher B1 ou B2. Trata-se, nesse sentido, de uma ambiguidade determinada pelas relações treinadas e pelo contexto das tarefas de teste. Interessante, a esse respeito, foram as escolhas, em tarefas como essa, realizadas em favor do terceiro estímulo (B3, por exemplo) quando não há desempenho de treino que seja coerente com esse tipo de resposta de escolha, conforme as relações de treino. Mesmo assim, apenas três dos 10 participantes apresentaram resultados inconsistentes com a formação de classes. É importante lembrar que apenas um dos quatro participantes do Experimento 2 apresentou esse tipo de variabilidade nos resultados de emergência, o que pode indicar a importância 
da revisão das relações treinadas. Sugere-se, a esse respeito, que novas investigações sejam realizadas a fim de se verificar o papel que a alternativa não envolvida com a ambiguidade na formação das classes tem em situações de conflito, como aquela presente nos dois experimentos aqui relatados.

Finalmente, a interpretação comportamental da emergência atrelada à formação de classes de estímulos equivalentes, formalizada pela proposição de Sidman e Tailby (1982) tem sido inquestionavelmente produtiva, seja na formulação de procedimentos experimentais eficientes na obtenção de informações empíricas decisivas para a área, seja na interpretação do comportamento simbólico em geral, mas a identificação das suas limitações pode desempenhar um passo importante para o avanço da área. A linha de investigação impulsionada por Pilgrim e Galizio (1995), e na qual se insere o presente estudo, tem sido muito produtiva nessa identificação. Por exemplo, nem todas as relações simbólicas presentes na vida das pessoas obedecem à linearidade de relações como A1B1, B1C1, A1C1 etc. Os símbolos empregados em situações comuns do dia a dia são na maior parte das vezes polissêmicos e, em parcela importante, também ambíguos. Nesse sentido, os resultados do presente estudo podem colaborar com a busca de novas interpretações e propostas de investigação do comportamento simbólico, em especial que visem incluir a sobreposição de classes equivalentes.

\section{REFERÊNCIAS}

Almeida, J. H., \& Haydu, V. B. (2011). Reorganization of equivalence classes: Analysis of reversed baseline relations. Psicologia: Reflexão e Crítica, 24(3), 609-620.

Bortoloti, R., Rodrigues, N. C., Cortez, M. D., Pimentel, N., \& de Rose, J. C. (2013). Overtraining increases the strength of equivalence stimulus. Psychology \& Neuroscience, 6(3), 357-364.

Carvalho, M. P., \& de Rose, J. C. (2014). Understanding racial attitudes through the stimulus equivalence paradigm. The Psychological Record, 64(3), 527-536.

Dickins, D. W., Bentall, R. P., \& Smith, A. B. (1993). The role of individual stimulus names in the emergence of equivalence relations: The effects of paired-associates training between names. The Psychological Record, 43, 713-724.

Dickins, T. E., \& Dickins, D. W. (2001). Symbols, stimulus equivalence and the origins of language. Behavior and Philosophy, 29, 221-244.

Doran, E., \& Fields, L. (2012). All stimuli are equal, but some are more equal than others: Measuring relational preferences within an equivalence class. Journal of the Experimental Analysis of Behavior, 98, 243-256.

Ferreira, P. R. S., Domeniconi, C., \& de Rose, J. C. C. (2010). As extensões do tacto segundo a concepção de propriedade de estímulo. Acta Comportamentalia, 18, 257-278.

Fields, L., Adams, B. J., Verhave, T., \& Newman, S. (1990). The effects of nodality on the formation of equivalence classes. Journal of the Experimental Analysis of Behavior, 53(3), 345358. http://doi.org/10.1901/jeab.1990.53-345

Fields, L., Arntzen, E., Nartey, R. K., \& Eilifsen, C. (2012). Effects of a meaningful a discriminative, and a meaningless stimulus on equivalence class formation. Journal of the Experimental Analysis of Behavior, 97, 163-181.

Folsta, A. G., \& de Rose, J. C. (2007). Rearrangement of equivalence classes after reversal of a single baseline relation: Influence of class size. Experimental Analysis of Human Behavior Bulletin, $25,1-5$.

Garotti, M., \& de Rose, J. C. (2007). Reorganization of equivalence classes: Evidence for contextual control by baseline reviews before probes. The Psychological Record, 57, 87-102.

Garotti, M., de Souza, D. G., de Rose, J. C., Molina, R. C., \& Gil, M. S. A. (2000). Reorganization of equivalence classes after reversal of baseline relations. The Psychological Record, 40, $35-48$.
Mackay, H. A., Wilkinson, K. M., Farrell, C., \& Serna, R.W. (2011). Evaluating merger and intersection of equivalence classes with one member in common. Journal of the Experimental Analysis of Behavior, 96, 87-105.

Nevin, J. A. (1992). An integrative model for the study of behavioral momentum. Journal of the Experimental Analysis of Behavior, 57, 301-316.

Pilgrim, C., \& Galizio, M. (1995). Reversal of baseline relations and stimulus equivalence: I Adults. Journal of the Experimental Analysis of Behavior, 63, 225-238.

Pilgrim, C., Chambers, L., \& Galizio, M. (1995). Reversal of baseline relations and stimulus equivalence: II Children. Journal of the Experimental Analysis of Behavior, 63, 230254.

Saunders, R. R., \& Green, G. (1999). A discrimination analysis of training-structure effects on stimulus equivalence outcomes. Journal of the Experimental Analysis of Behavior, 72, 117-137.

Sidman, M. (1971). Reading and auditory-visual equivalences. Journal of Speech and Hearing Research, 14, 5-13.

Sidman, M. (2000). Equivalence relations and the reinforcement contingency. Journal of Experimental Analysis of Behavior, 74, 127-146.

Sidman, M., Cresson, O., Jr., \& Willson-Moris, M. (1974). Acquisition of matching-to-sample via mediated transfer. Journal of the Experimental Analysis of Behavior, 22, 261-273.

Sidman, M., Rauzin, R., Lazar, R., Cunningham, S., Tailby, W., \& Carrigan, P. (1982). A search for symmetry in the conditional discriminations of rhesus monkeys, baboons and children. Journal of the Experimental Analysis of Behavior, 37, 23-44.

Sidman, M., \& Tailby, W. (1982). Conditional discriminations vs. matching-to-sample: An expansion of the testing paradigm. Journal of the Experimental Analysis of Behavior, 37, 5-22.

Skinner, B. F. (1957). Verbal behavior. Englewood Cliffs, NJ: Prentice-Hall.

Smith, A. B., Dickins, D. W., \& Bentall, R. P. (1996). The role of individual stimulus names in the emergence of equivalence relations II: The effects of interfering tasks prior to and after tests for emergent relations. The Psychological Record, 46, 109-130.

Spradlin, J. E., Cotter, V. W., \& Baxley, N. (1973). Establishing a conditional discrimination without direct training: A study of transfer with retarded adolescents. American Journal of Mental Deficiency, 77, 556-556. 